\title{
Techno-Creativity: The Implementation of DVFx as Creative Support Tools Among Filmmakers in Malaysia, India \& Australia
}

\author{
HASRUL HASHIM \\ Universiti Kebangsaan Malaysia
}

\begin{abstract}
Previously, the use of digital visual effects (DVFx) only serves merely to fix or modify the damage or harmful elements in the post-production. Still, there are differences between improving the work and the creative effects in work. Subtracting part of the image can often be seen as a form of creativity in generating new image elements. DVFx is also seen as a form of creative effects. It is a fascinating field of study on 'adverse impact' or 'creative impact' in film production. Creative impact in the production process of film is likely to lead to a new assessment on the aspects of 'creativity.' The occurrence of technological changes that involve creative work will undoubtedly lead to a shift in creativity. Technology and creativity or techno-creativity are likely to represent a new creative possibility and measurement in film production using creative software as a support tool. This paper discusses the findings derived from in-depth interviews conducted with filmmakers, visual effects artists and supervisors from Malaysia, India, and Australia on implementing DVFx as creative support tools. The finding shows that DVFx functions as a techno-creative device in two main areas: ideation and implementation. These two aspects involve using DVFx as a tool for transforming the ideas and the imagination of a filmmaker into a reality on the screen. In other words, DVFx has acted as creative support tools (CST) to increase techno-creativity, especially in the area of visual presentation.
\end{abstract}

Keywords: Creativity, digital visual effects, creative support tools, media technology, globalization.

\section{INTRODUCTION}

Before discussing cinema technology development, it is preferable to know much about the world's film industry historical strains to understand the relationship between technology and cinema. Throughout the history of cinema, it will also provide a possible understanding of that impatient viewer and the interactive user (Elsaesser, 2015) because technology and cinema significantly connect to the audience. In 1895, the Lumiere brothers, for the first time, used the cinematograph motion picture camera and presented the Salon Indien du Grand Café in Paris. Apart from creating the cinematographers, there were also technological inventions of motion pictures, such as Joly's cinematograph, the Biograph, and Gaumont's 60mm motion picture system (Musser, 2018). At the beginning of the cinema industry, the cinematograph's invention was seen as a spark of the new technology or 'eureka moment.' This technological system of projected motion pictures has evolved to screen in a theatrical setting and involves profit and economy aspects. The audience is willing to pay to experience the cinema spectacles.

The main question highlighted in this study concerns the impact of DVFx technology used in the film industry. As such, this situation is related to ontology and debates over technological determinism. In essence, the concept that has been founded by Marshall McLuhan posits that technological development determines cultural and social change (McLuhan, 1964). McLuhan believes that humans adapt to the environment and the development of technology through a 
precise balance of their senses, and the medium of the age brings out the ratio. This view indirectly proves that the DVFx revolution in the film industry positively impacts the creativity and narrative of a film.

The idea of technological determinism has led to an intellectual debate among communication scholars. Marx and Smith (1994) argue that technological power is an agent of social change that has a very prominent place in the culture of modernity. According to them, the idea of technological determinism exists on two spectrums between 'hard' and 'soft' change. In the 'hard' spectrum, agency (which refers to the power of change) imputed the technology itself. Acceptance of technological advancement is inevitable based on particular needs. In the hard determinist's future vision, technology has been embraced in life whether for good or bad, because technologies permit few alternatives. The 'soft' spectrum begins with the understanding that the history of technology is the history of human progress. In addition to turning technology into a historical agency, the soft determinists put it far beyond the social, economic, and cultural aspects of culture. The soft determinist view of technology is influenced by the way they might explain the growing credence. Thus, both the technological determinists of the 'hard' and 'soft' spectrum are deeply embedded in the social structure and culture to divest the technology as the agent of change.

Digital visual effects (DVFx) have revolutionised creative works' consumption, enabling and refreshing new forms of distribution and transforming the interaction with the audience. Indirectly, today's technology has also brought a unique perspective to the audience taste, including the usage and type of content (Asma Isa, Wan Amizah \& Wan Idros, 2020). It also changes the definition of 'creativity'. Through digital cameras, mobile devices, apps and software, creativity in film productions has entered a new phase. Technology-based creativity has also brought a new culture to film studies and the field of communication. According to Rakhe (2012), techno-creativity studies refer to an intensive study of various theories, campaigns and views of creative people and their reaction to technology-based creativity. Rakhe uses the term technocreativity to refer to the use of the most advanced technology in advertising to creatively communicate to the masses, such as using 3D graphics to shoot high-definition commercial videos. From another perspective, the globalisation of technology through the advent of DVFX needs to be seen as a new opportunity for industry players to enhance visual creativity and storytelling in films using DVFx as a creative support tool (CST). DVFx is also seen as a medium that crosses geographic space, sharing technology and expertise. Therefore, this research aims to study the impact of DVFx on creativity and serves as a creative support tool of 'technocreativity' amongst the film industry's creative players in Malaysia, India and Australia. A comparative case study on the use of DVFx as techno-creativity among these countries can provide a new dimension and a new formula for cross-border production, sharing technologies and creativity. Put simply; this paper suggests a suitable space for institutions and individuals to form valuable connections between experts in digital visual representation in those countries.

\section{DVFx and The System Views of Creativity}

Hennessey (2015) notes that the vast majority of researchers in creativity focus on the creative process, the creative person and the innovative product, but give less attention to the creative milieu. The creativity area will have to be more developed before investigators can develop a 
systems view of creativity. Creativity is not bound to any single discipline. Practically all fields see creativity in their way. According to Runco and Jaeger (2012), previous scholars have used different criteria to define creativity. There are two main criteria used in determining creativity, which is originality and effectiveness. Ingenuity is undoubtedly required, and it is often labelled novelty; meanwhile, there are other fundamental requirements for creativity. Effectiveness may take the form of value. This describes how original and valuable products and ideas depend on the current market, and more specifically, on the cost and benefits of defying the trend.

Csikszentmihalyi (1999) is among the pioneers who created the systems view of creativity, which is used in various disciplines, including the film industry and creative industries. The three small systems introduced by Csikszentmihalyi (domain, field and individual) are very closely related to cultural contexts that will determine other domains such as knowledge, tools, values and practice, which are also driving elements of the creative process (Hennessey, 2015). Essential concepts such as novelty and value provide a better understanding of creativity in this field. Csikszentmihalyi's systems view implies that creativity cannot be understood by isolating individuals and their work from their social and historical surroundings.

Redvall (2012) has proposed a new framework for film case studies and media production using Mihaly Csikszentmihalyi's systems view of creativity. Redvall recommends a combination of Csikszentmihalyi's model and a creative process model from the school of creative problem solving (CPS). Nevertheless, the model proposed by Redvall is only suitable for analysing creativity during the production process. Through analysis at various stages of production, researchers can see how identifying problems and solving problems can help create creative, novel and high-quality work.

The creativity of production, especially the production of a film, is necessarily assisted by support tools and technology. Creative support tools (CST) are any tool that any individual can use to produce any product that involves creativity. It is not solely focused on film production or DVFx. In other words, CST is a complementary agent to the aspect of creativity based on technology or techno-creativity. CST can exist in any creative areas such as craftsmanship and manufacturing, visual arts, voice art, music et cetera but in the field of digital technology such as computing, CST exists in the form of software applications to create digital art such as animation and visual effects. For example, a visual effects artist will use animation software such as Flash, Virtual or Adobe Illustrator as a CST to produce a scene that requires a digital visual effect.

According to Cherry and Latulipe (2014), CST is a diversity of equipment or tools that help humans connect creativity to the world. One example of equipment or devices used frequently in DVFx production is the computer. According to Fink and Morie (2014), a computer is a powerful tool or powerful CST used to produce fantastic images, but its use needs to be combined with the vision of a DVFx artist. CST can also impact individuals or communities by improving areas of expertise such as science, engineering, humanity and arts. CST also can influence creative work as well as its ability to bring about rare creative moments that can impact the community.

\section{METHODOLOGY}

The selection of Malaysia, India and Australia as the primary research sample and subjects was based on the rationale that these three countries represent different landscapes and abilities in creativity and DVFx technological advancement in their national cinemas. While these three 
countries have various capacities, this will allow comparisons that highlight DVFx's potential to be a tool for techno-creativity. In-depth interviews were conducted with 21 experts in the film and visual effects industries of the three countries. Geographical boundaries and distances have opened up space for researchers to integrate social media technology or digital platforms in the sample search process.

Therefore, in this study, informants were sought through the Linkedln professional network application. Linkedln is a professional network service that provides profiles of professionals worldwide and encompasses various fields of work, including the DVFx area. Through this social media application directors, producers, DVFx supervisors and DVFx artists were invited to be informants in this study based on their profile and experience in the field of film and DVFx technology. The informants' expertise and work in the film industry were also confirmed through the IMDb website. The Internet Movie Database (IMDb) is a worldwide online database that provides information related to movie ratings, production crews, and director biographies and actors involved in films.

Most of the informants involved in this study were DVFx supervisors, DVFx artists, producers, film directors or academics. After identifying a potential informant, I contacted them via email and sent them a participant information form (PIF) to gain a clear and bigger picture of the research project. Further informants were then found through snowball sampling based on mutual connections in the initial informants' profiles and suggestions from the informants. This process involved the integration of conventional snowball methods as well as digital platform methods. More than 75 informants were approached via Linkedln, but only 35 informants expressed their interest. Finally, out of this amount, only 21 informants gave consent to be informants. To ensure that the selected informants were suitably qualified and of high calibre, the informants in this in-depth interview process were required to meet the following criteria:

i. Informants must comprise either film directors, film producers, DVFx supervisors, DVFX artists or academics in the film area with at least three years of experience. This experience factor is vital to ensure the breadth and depth of data and information to be acquired.

ii. Informants have been involved in films using DVFx technology in the film industry in Malaysia, India or Australia. This criterion means that informant citizenship is not a significant factor that would prevent them from engaging in this study.

iii. Informants from the industry (directors, DVFx supervisors and DVFx artists) must have been involved in at least three films using DVFx regardless of whether they were local or international. This factor is to ensure that informants have the right capability and reliable experience in this area. 


\section{DVFx as Creative Support Tools}

\section{FINDING}

Table 1: Examples of CST Software

\begin{tabular}{lll}
\hline Country & Category & Example of CST software \\
\hline Malaysia & Shooting & Chromakey \\
& Tracking & Bojo, Screen Touch, Scene Eye \\
& 2D \& 3D & After Effects, 3D Photoshop, Illustrator, 3D Max \\
\multirow{5}{*}{ India } & Compositing \& editing & After Effects, Mocca, Fusion, Final Cut Pro \\
& Modelling & Bojo, After Effects, Illustrator \\
& 3D & Maya, VDay, Katana, Claris, 3D Max \\
& Compositing \& editing & Final Cut Pro, Nuke, Flame \\
& Storyboarding & ToonBoom \\
& 2D & After Effects, Photoshop, Animate, Adobe Premiere \\
& 3D & Autodesk Maya, 3DsMax, Cinema 4D, Nuke, Houdini, \\
& & Autodesk Flame, Katana, RealFLow, Blender, Unreal \\
& & Engine 4, Yeti \\
& Compositing \& editing & Media 100 Editor, After Effects \\
\hline
\end{tabular}

Based on the findings, almost all the informants in the DVFx industry used the same CST to produce their respective visual effects projects. Arguably, the software used by the visual effects artists from these three countries is used by most professional visual effects companies worldwide. Table 1 shows examples of CST software in a movie production project that uses DVFx. Based on the table, informants from Australia and India provided a more comprehensive software list than informants from Malaysia. This is probably because the projects carried out by the DVFx companies in Australia and India are more international than in Malaysia, which is characterised by the local market. However, informants from all countries listed a universal CST software simulation that is used globally.

Based on the findings, a DVFx artist must know how to use CST to facilitate the creative process. The informants stated that if a DVFx artist knows how to use any CST software, they should have no problem using other software. AUS5 explained this:

Things like the advance of technology are just make intend more accessible for the artist to focus on the creative side and not worry too much on the technical side. You still need to know how to use the software, but it is getting easier to pick up the new software nowadays, as long as I know the fundamentals of how modelling things works.

However, this is a bit different from the situation among Malaysian informants. According to informant MAS1, using the software beyond one's field of expertise is difficult and requires time to study. MAS1 said:

It would be hard to learn to use the software, except it is out of your capability. For example, I am not very familiar with $3 \mathrm{ds}$ Max, and it takes time for me to learn that even I have a basic knowledge of 3D. 
MA2 shared the same situation. According to MA2:

I'm using Photoshop for CGI and Illustrator for 2D elements. I'm using After Effects as well for composition and Scene Eye for tracking. I am not familiar with the 3D software because we have our expertise, so since I am a compositor and clean up artist, I'm only familiar with After Effects, Bojo and Mocca.

Nevertheless, the specialisation of assignments and expertise practised by Malaysian informants is, in fact, not unusual. According to an informant from India, IND1, speciality assignments and expertise were practised in India to meet customers' demands and the layout of pipeline production. The production pipeline refers to the stages of the process of producing a film. Each step of the process requires different tools. For example, in the production of a scene requiring $\mathrm{CGI}$, the production stage will be divided into the modelling, tracking, 3D, compositing and subsequent rendering processes. Each of these levels requires different software. As such, the work is breaking down in the process according to the pipeline. IND1 explained this:

Different companies are using different kinds of software. Some of them using Maya, some of them using Max, some of them using Claris, are very unpredictable. It does all depend on what kind of pipeline that industries will follow it.

Based on these findings, specialisation and expertise in using CST is familiar but still contribute to creators' creativity. In producing DVFx creative works, CST is just a support factor for the creativity within the individual or group concerned. Depending on CST, creating a work does not guarantee a genuinely creative product that is acceptable to an audience. On the contrary, natural creativity within the individual is still necessary. One informant agreed that CST is only a support tool informing or influencing the creativity of an artist's visual effects, and the rest is dependent on the individual's attitudes, efforts and personality. The informant from Malaysia, MAS2, explained:

I used to learn all this software since university but only the basics. If you want more, you need to explore by yourself. I always refer to YouTube for tutorials and online forums to look for new software and suggestions from other users. I might say that $70 \%$ of my VFX knowledge came from experiences and myself. We should explore to gain more.

IND4 agreed with this:

Software is always easy, so implementation is still an important thing because if you know the shots' ethics and aesthetics, you know what is done. As long as your aesthetics are very clear about it, that theory is prominent, and the implementation of those things are practical. The software will not be 
complicated to use. Software is just giving you a tool, but in the end, you are the artist.

This suggests that the integration between natural creativity and knowledge of technology is the best formula for improving the quality of visual performances within DVFx. These findings are in line with Fink and Morie's (2014) argument that even though technology or CST is an excellent tool in helping a DVFx artist, imagination and innovation in the individual who uses the equipment to create a whole new fantasy world is the key to creativity.

\section{DVFx as a Measure of Techno-creativity}

Findings from the informants showed two different sides of this issue. Informants from Malaysia and Australia believe that technology-based creativity assessment or techno-creativity using DVFx is imperative and were used as one of the tools to measure creativity. Informants from India say there are difficulties in doing so and they think that evaluating creativity is difficult since it is subjective. Informant IND1 holds that:

I don't think visual effects can determine how creative you are. I think it's the person using the software/tools. It's all about how you use those tools and what you can give to the original.

IND1 provided an example of someone who has been in the industry for 60 years and is not familiar with CST or DVFx software than someone who is 15-years-old but knows how to use CST and modern software. IND1 added:

So both of them making a film using visual effects will become perfect. So how do we define who is the most creative? Is it someone who is 60 years in the industry is still making new idea, new ideas, or is it a 15-years-old who suddenly got hands-on with the software and making something?

This opinion is valid but is considered in the context of measuring creativity. For IND2:

There is no scale to measure how much creativity. Creativity is like infinite. You can do anything about it with your imagination. DVFx is just a tool to manipulate things or create things. Otherwise, it's just imagination on how you want to show the items.

These two informants are trying to show that art and creative values derived from the individual themselves, and the technology or CST is a helpful element. The second point of view is that DVFx were used in the production process as a measuring stick or instrument in assessing creativity, especially the creativity of a film produced. According to informant MAS1, some films look great due to the DVFx: 
If you think about the use of visual effects, if you watch a short film, for example, Transformers, I know that kind of genre is about spectacle. It's about the visual of it. So the audience would measure the creativity, how creative it is, by looking at the technology created by that particular special effects.

MAS1 added:

Even Lord of the Rings, for example, people measure creativity, 'wow, very creative', because of the images they have not seen before, they have not thought of before, or they have a very vague idea about it kind of concretised. So, in the sense that when you look at CGI, for example, as a way for people to see 'wow, it is creativity' when it creates that 'wow', that wonder. That sense of wonder means the film has achieved something.

An informant from Australia, AUS4, stated that some films creatively presented through the use of DVFx, which supports the opinion of MAS1. According to AUS4:

Indeed, visual effects open up the scope of what is capable of storytelling. So there is a lot of stories that just wouldn't exist without visual effects. Look at Stephen Spielberg's latest film, 'Ready Player One. You could never have that film, could never have made ... essentially the animated film, you could never have told the story creatively.

Informant AUS2 argued that the DVFx user is considered creative as DFVx artists create the entire object and subject in some produced film projects. According to AUS2:

It is creativity because I think many films these days are just an actor, or a green screen or blue screen and nothing else. So we have to create that whole world. I think in 'Great Wall' a lot of the shots were entirely CG.

AUS1 supported this opinion. According to AUS1, using CST software in producing DVFx is innovative and unique as there is software that opens up space to be creative. AUS1 said:

I think the way people use the programs can be innovative and unique, and they created some fascinating stuff, but I also think it depends on the software. Software like Maya, while it does allow for creativity to come forth and create something different or innovative or unique, can also be used by people who are less creative and more technical minded creating something spectacular.

According to MAS2, creativity was measured based on the use of technology. According to him, the use of technology, especially DVFx, can be used as an instrument in measuring and assessing creativity: 
When digital technology came in, I think it helps our industry a lot because it jump-started lessons. Many people skip the most crucial part of filmmaking because we do not have enough skills and expertise. So when the digital technology came in we could go to that level five without going to two, three, four ... So it was a bit helpful in that sense.

Despite this, the views of MAS2 are slightly different from the views expressed by informant AUS3. Informant AUS3 agreed that there is various CST software in the market due to the rapid development of technology, but this development is due to the industry. According to AUS3:

I do not think the issue is in word creativity. I do not think the problem is the development of the software. I think it will be led by the industry's issue and where the money is, and if the money is not to save free-to-air TV, the money is no longer in theatrical films. Then the creative tools will move to match whatever the industry demands.

In conclusion, DVFx, as one of many tools were used to measure the creativity level of a film through two aspects of the audience evaluation, alongside the use of software or CST technology. While creativity is infinite, technological developments in the film industry have changed the film production landscape more than any other factor; technologies such as DVFx make impossible things possible in the real world.

\section{DISCUSSION}

The film industry developments show that DVFx has had a significant impact on narratives and creativity over the decades. This impact can be seen within changes in narrative presentation techniques and the use of tools in production. The analysis in this section begins by looking at what factors cause the informants to use DVFx to produce their movie projects. This analysis is essential to know the extent to which professionals in these three countries have adapted to cyberculture, as described by Alexander (2017). Simultaneously, the analysis of the use of this technology also coincides with the concept of affordance to investigate at what stage people learn to use 'action possibilities' or affordances in their creative environment (Shaw, 2017). An affordance is a conceptual tool frequently used by scholars in the social science of technology and communications. The use of the term affordance has grown widely in this area as it helps to explain the implications of technology for people. Affordance refers to possibilities in how objects in the environment could be used. Researchers in communication technology studies use this term to explain the impact of technologies on people (Shaw, 2017).

The technical aspects of film production are also an important aspect to be highlighted in film studies (Nurul Ezzati \& Wan Hartini, 2020). Numerous previous studies have focused on defining and evaluating creativity, but research involving creativity and technology and explored CST is a new field. One of the studies conducted by Cherry and Latulipe (2014) focused on assessing creativity and creative support tools (CST). However, in evaluating CST, it should be noted that there is no one-size-fits-all approach that researchers can use. Because of this, 
researchers need to use a variety of resources and metrics to demonstrate the validity of their measurements. Most of the definitions of creativity focus on producing something that is considered new, so we forget that the basic concepts that are the heart of creativity are 'novelty' and 'value'. Measuring creativity is very subjective. In addition to the difficulty of predicting creativity, it is also challenging to interpret and measure (Cherry \& Latulipe, 2014).

The analysis has also shown that techno-creativity involves the concepts of ideation and implementation. These concepts were seen as parts of a single process, but they are very different. Creativity exists when it combines the function of cultivation and expansion of ideas before being implemented to make a visible end-product. Hence, the process of a film must follow this process. Therefore, I will use these two concepts of ideation and implementation to interpret techno-creativity ideas further.

In the concept of techno-creativity used in this study, I propose the concept of ideation, which refers to the natural creativity inherent in individuals and not assisted by any tools that strengthen creativity. In comparison, the idea of implementation refers to the technology or tools used by the individual to express their creativity in a tangible form. Ideation and implementation interact through both talent and tools through technology assistance such as CST (refer to Figure 1). For a more explicit example, referring to this study, techno-creativity is a creative process in which a DVFx artist implements technology to create digital special effects scenes in a film, which indirectly helps develop the story, narrative and visual presentation.

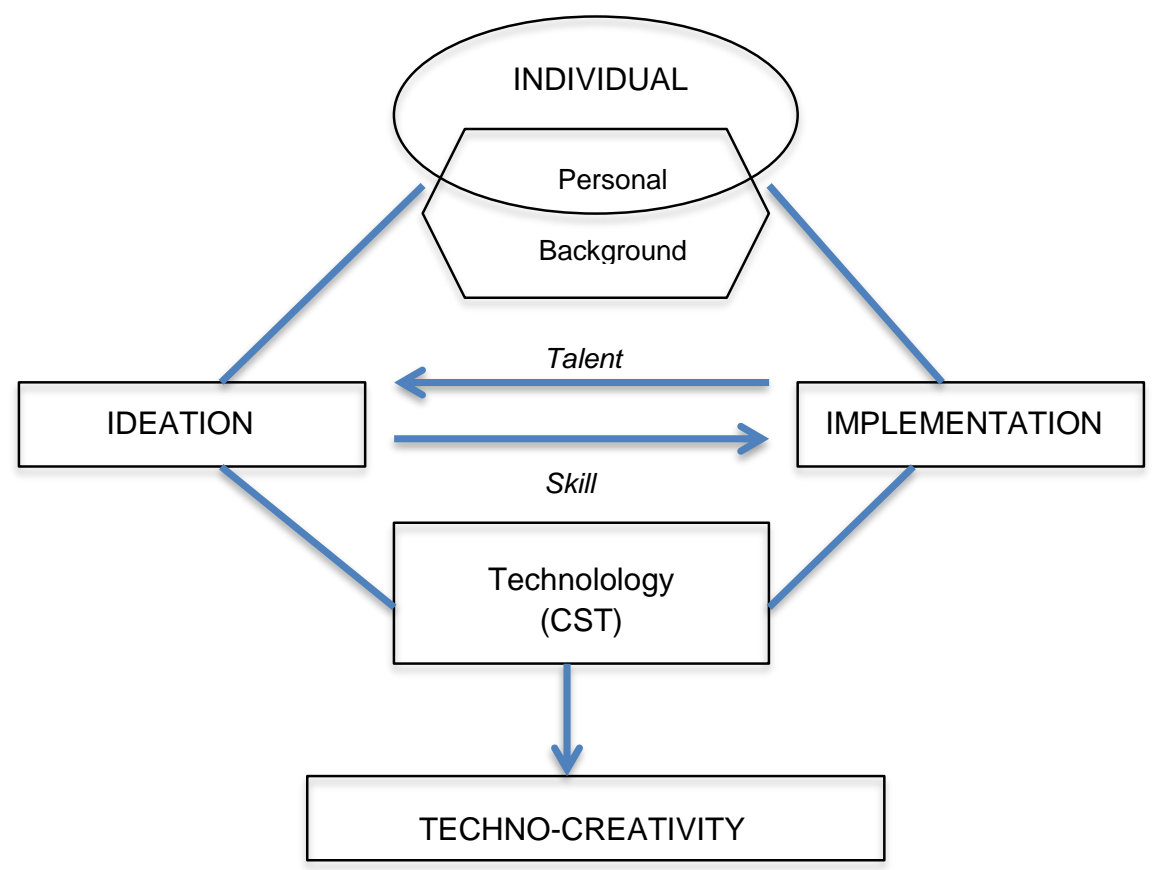

Figure 1: The Systems View of Techno-Creativity 
Creativity is often associated with newness, but in reality, the convention usually shaped it. It is about transforming the material by referring to the existing rules, devices, codes, and procedures. Creativity in media and cultural production involves the conflict between industry (commerce) and creativity among artists, producers, and directors. From another angle, marketing and creativity have been mixed and bound together in the modern economy. Thus, this situation has indirectly 'created' the creative industry because the businesses involved in cultural productions do not possess any form of criteria or guidelines for harnessing creative practices. However, creativity is not just a descriptive term; it also provides a sense of value and establishes a cultural hierarchy. Today, we are moving towards seeing technology as necessary in many work areas, including creativity in the film and creative industries. Day by day, creative employees in this field introduce a new culture in the visual presentation using existing technologies such as DVFx. Not all creative individuals can use technology and adapt to innovation, as purchasing and learning the technologies is expensive. The findings in this study show that the creativity inherent in the system has been integrated through the emergence of technology, especially in the film industry. DVFx is one of the forms of technology inherent in using CST combinations, which should be part of a definition of creativity based on technology or techno-creativity.

The findings also show that concepts such as techno-creativity can be a useful tool for analysing both film and media production to understand the overall operation of the creativity system (Redvall, 2012). Consequently, in conclusion, the most critical aspect of technocreativity in film production is the ability to produce quality work and at the same time help with the narrative and visualisation process of the story so that the message communicated to the audience. Technologies such as DVFx should be compatible with the message they convey rather than just a trend or a commodity.

\section{CONCLUSION}

The discussion on film aesthetics and cinematic representation should be discussed from multiple points of views (Mohd Erman, Mastura Muhammad \& Harith Baharuddin, 2020). Technocreativity can also be used as a measure to analyse and understand the creative aspect of the film and other media productions based on the overall operation of the systems view of creativity. Techno-creativity among creative industry players also engages the appeal of digital humanity. These findings are consistent with Muller and Ulrich (2013) argument that the critical aspect to be given attention in this area of creativity is the clashes between two different identities, which are humanity and technology. Also, this finding is in line with Miller and Sinanan's (2014) theory of attainment, which states that technology should not change humanity while making us more human. Technology should serve as a tool and opportunity that can foster a creative industry that can positively react to technology affordance.

It has been stated earlier that most of the scholarly studies on CST have shown a connection between the use of CTS and the level of creativity. Similar findings also emerged from this study, which showed that most informants agree that CST serves as a tool in enhancing a person's creativity. Furthermore, informants agree that human factors such as skills and knowledge are also essential factors in techno-creativity. While CST is a tool for developing an artist's or users' creativity, there is a relationship between the support tools used and the devices' 
complexity. A fun tool is likely to be easy to learn and useful for simple tasks, but it is expected not to have 'power' to support the complex tasks required in creative work such as DVFX production. Hence, in this respect, the expertise and skills in the individual CST users play a significant role in integrating technology and creativity, or techno-creativity.

According to Becker (1982), producing artistic work involves a different variety of activities and a large number of people. Therefore, the work shows a sign of cooperation. Becker (1982) also argues that this form of collaboration may be ephemeral but often produces collective activity patterns in the art world. He has also emphasised the ways that cultural texts are created through structured collective action. This 'structured' action is related to the three main systems of individual, domain and field in the system view of creativity, to explain how they operate in the DVFx industry. Based on interviews and previous studies, the technological revolution, especially DVFx, has impacted the process of enhancing creativity. The analysis shows that DVFx technology has transformed human experiences to lead to techno-creativity in the film industry, including in Malaysia, India and Australia, through the use of creative support tools (CST) and the influence of social environments such as audience demand and market supply.

BIODATA

Hasrul Hashim is a senior lecturer at the Centre for Research in Media \& Communication, Faculty of Social Sciences \& Humanities, Universiti Kebangsaan Malaysia. Email: hash@ukm.edu.my 


\section{REFERENCES}

Asma Md Isa, Wan Amizah Wan Mahmud, \& Wan Idros Wan Sulaiman. (2020). The combining of intrinsic and extrinsic motives for employing OTT media and comprehending the audience's gratification in Malaysia. Jurnal Komunikasi: Malaysian Journal of Communication, 36(3), 266-280.

Alexander, B. (2017). The new digital storytelling: Creating narratives with new media. Praeger.

Becker, H. S. (1982). Art world. University of California Press.

Cherry, E., \& Latulipe, C. (2014). Quantifying the creativity support of digital tools through the creativity support index. ACM Transactions on Computer-Human Interaction, 21(4), 1-25.

Csikszentmihalyi, M. (1999). Handbook of creativity. Cambridge University Press.

Fink, M., \& Morie, J. F. (2014). Introduction. In J. A. Okun \& S. Zwerman (Eds.), The VES handbook of visual effects: Industry standard VFX practices and procedures (pp. 1-15). Routledge.

Hennessey, B. A. (2015). Creative behaviour, motivation, environment and culture: The building of a system model. Journal of Creative Behavior, 49(3), 194-210.

Marx, L. \& Smith, M. R. (1994). Does technology drive history? The dilemma of technological determinism. MIT Press.

Miller, D., \& Sinanan, J. (2014). Webcam. Polity Press.

Mohd Erman Maharam, Mastura Muhammad, \& Harith Baharuddin. (2020). Sinema nasional Malaysia: Pasang surut budaya dalam tiga era. Jurnal Komunikasi: Malaysian Journal of Communication, 36(3), 228-246.

Muller, S., \& Ulrich, F. (2013). Creativity and information systems in a hypercompetitive environment: A literature review. Communications of the Association for Information Systems, 32(7), 175-200.

Musser, C. (2018). When did the cinema become cinema? Technology, history and the moving picture. In Hidalgo, S. (Ed.), Technology and film scholarship. Amsterdam University Press.

Nurul Ezzati Aisya Mohd Zaki, \& Wan Hartini Wan Zainodin (2020). P. Ramlee's cinematographic Auteurship of cultural value representation in Antara Dua Darjat and Ibu Mertuaku. Jurnal Komunikasi: Malaysian Journal of Communication, 36(3), 390-407.

Rakhe, S. (2012). Techno creativity in advertising [PhD dissertation]. ProQuest Dissertations Publishing (3736032).

Redvall, E. N. (2012). A systems view of film-making as a creative practice. Northern Lights: Film \& Media Studies Yearbook, 10(1), 57-73.

Runco, M. A., \& Jaeger, G. J. (2012). The standard definition of creativity. Creativity Research Journal, 24(1), 92-96.

Shaw, A. (2017). Encoding and decoding affordance: Stuart Hall and interactive media technologies. Media, Culture \& Society, 39(4), 592-602. 